\title{
Study of Gaussian Relay Channels with Correlated Noises
}

\author{
Lili Zhang, Jinhua Jiang, Andrea J. Goldsmith, and Shuguang Cui
}

\begin{abstract}
In this paper, we consider full-duplex and half-duplex Gaussian relay channels where the noises at the relay and destination are arbitrarily correlated. We first derive the capacity upper bound and the achievable rates with three existing schemes: Decode-and-Forward (DF), Compress-and-Forward (CF), and Amplify-andForward (AF). We present two capacity results under specific noise correlation coefficients, one being achieved by DF and the other being achieved by direct link transmission (or a special case of CF). The channel for the former capacity result is equivalent to the traditional Gaussian degraded relay channel and the latter corresponds to the Gaussian reversely-degraded relay channel. For CF and AF schemes, we show that their achievable rates are strictly decreasing functions over the negative correlation coefficient. Through numerical comparisons under different channel settings, we observe that although DF completely disregards the noise correlation while the other two can potentially exploit such extra information, none of the three relay schemes always outperforms the others over different correlation coefficients. Moreover, the exploitation of noise correlation by $\mathrm{CF}$ and AF accrues more benefit when the source-relay link is weak. This paper also considers the optimal power allocation problem under the correlated-noise channel setting. With individual power constraints at the relay and the source, it is shown that the relay should use all its available power to maximize the achievable rates under any correlation coefficient. With a total power constraint across the source and the relay, the achievable rates are proved to be concave functions over the power allocation factor for $\mathrm{AF}$ and $\mathrm{CF}$ under full-duplex mode, where the closed-form power allocation strategy is derived.
\end{abstract}

\section{Index Terms}

Capacity Bounds, Relay Channel, Correlated Noises, Power Allocation.

\section{INTRODUCTION}

The relay channel was first introduced by van der Meulen [1], in which the source communicates with the destination via the help of a relay node. It was then thoroughly studied in [2], where a max-flow min-cut upper bound and three achievable rates have been developed for the channel. Among the three achievable rates, two were obtained with the well-known relaying strategies: Decode-and-Forward (DF) and Compressand-Forward (CF), respectively. The third achievable rate was obtained with a generalized coding strategy that 
combines the DF and CF strategies, which is known as the best achievable rate for the relay channel. Several known achievable rates meet the upper bound for a few relay channels of special configurations, leading to the corresponding capacity results. These channels include the degraded and reversely-degraded relay channels [2], the semi-deterministic relay channel [3], the phase fading relay channel [4], the orthogonal relay channel [5], and a class of deterministic channels [6]. Nevertheless, the capacity of a general relay channel is still an open problem.

In early works on the relay channel, the relay is usually assumed to be capable of operating in a full-duplex mode, i.e., the relay can transmit and receive signals at the same time over the same frequency band. This assumption may not be realistic due to practical restrictions such as the coupling of transmitted signals into the receiver path in the RF front-end. As such, more practical half-duplex relaying has been subsequently studied. In [7], the half-duplex frequency-division Additive White Gaussian Noise (AWGN) relay channel is investigated, where the channel is splitted into two frequency bands: One is utilized by both the source-relay and sourcedestination links, and the other is used by the relay-destination link. The counterpart time-division scheme is presented in [8], where at each slot the relay operates in the receiving mode for the first $\alpha$ fraction of time and in the transmitting mode for the remaining $1-\alpha$ fraction. The capacity upper bound and the achievable rates for the DF and CF schemes are derived in both frequency-division and time-division modes [7], [8]. Another much simpler relaying scheme under half-duplex mode, Amplify-and-Forward (AF), is discussed in [9], where the relay simply forwards a scaled version of the received signal in the time-division mode with $\alpha=0.5$.

Most of the existing relay channel work assumes uncorrelated noises at the relay and the destination. In this work, we investigate the effect of noise correlation on the Gaussian relay channel. This situation may arise in practice when the relay and the destination are interfered with by common random sources. We consider both the full-duplex and half-duplex relay channels under the correlated-noise assumption. We extend the max-flow min-cut upper bound and the DF, CF, and AF coding strategies to the relay channels with correlated noises. When applying the DF strategy, the relay decodes the information from the source, re-encodes and transmits it to the destination. Hence, DF completely disregards the correlation between the noises at the relay and the destination. In contrast, when applying the CF strategy, the relay compresses the received signal (including the noise) and forwards it to the destination as the side-information, which may enable us to exploit the noise correlation. Similarly, when AF is applied, the relay only forwards a scaled version of its received signal without decoding, and the destination processes the signals from the source and relay via Maximum-Ratio Combining (MRC), for which the noise correlation could also be exploited. Although both $\mathrm{CF}$ and AF appear to be more capable in exploiting the noise correlation, our numerical comparisons under various channel settings show that none of the coding strategies always outperforms the others across different correlation coefficients. Beyond the result of achievable rates, we discuss two capacity-achieving cases with special noise correlations where the achievable rates meet the upper bound, and they are in fact corresponding to the Gaussian degraded relay 
channel and the reversely-degraded one established in [2]. Moreover, it is shown that the noise correlation always helps with improving the $\mathrm{CF}$ and $\mathrm{AF}$ performance when $\rho_{z} \in[-1,0]$.

Based on the achievable rates for the relay channels with correlated noises, we also derive optimal power allocation policies under various constraints. We first consider an individual power constraint at the relay, and then the total power constraint across both the source and the relay. Under the individual power constraint at the relay, it is shown that the relay should always use all its power to obtain higher achievable rate under any noise correlations. With the total power constraint, we prove that the power allocation problems for the CF under full-duplex and AF schemes are both convex and yield unique solutions, and we provide several closed-form results for these solutions.

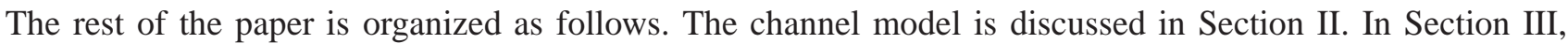
we provide the upper bound for the Gaussian relay channel with correlated noises under both full-duplex and half-duplex modes. The achievable rates and special capacity-achieving cases are presented in Section $\amalg$ The power allocation problems are studied in Section [V] Numerical results comparing different strategies against the capacity upper bound are presented in Section V. Finally, we conclude the paper in Section VI

\section{Channel Model}

Consider the Gaussian relay channel shown in Fig. 1, where the source conveys information to the destination with the help of a relay. The channels between the respective nodes are assumed to be experiencing static fading, and the link gains are denoted by $h_{21}, h_{31}$, and $h_{32}$, respectively. The noises that the relay and the destination suffer from are presumed to be arbitrarily correlated, possibly due to common interference sources. Under this correlated-noise channel setting, both full-duplex and half-duplex operating modes of the relay are investigated.

\section{A. Full-Duplex Mode}

In the full-duplex mode, the relay is capable of transmitting and receiving information at the same time over the same frequency band. In the $i$ th time slot, the channel inputs at the source and the relay are $X_{1}(i)$ and $X_{2}(i)$ with power $P_{1}$ and $P_{2}$, respectively. The relay experiences an AWGN noise $Z_{1}(i)$ with variance $N_{1}$, i.e., $Z_{1}(i) \sim \mathcal{N}\left(0, N_{1}\right)$. Similarly, the noise at the destination is denoted as $Z(i) \sim \mathcal{N}(0, N)$. We further assume that $Z_{1}$ and $Z$ are arbitrarily correlated, i.e., jointly Gaussian with correlation coefficient:

$$
\rho_{z}:=\frac{\mathbb{E}\left\{Z_{1} Z\right\}}{\sqrt{N_{1} N}} .
$$

Accordingly, the channel under full-duplex mode is fully described by the following two equations:

$$
\begin{aligned}
Y_{1}(i) & =h_{21} X_{1}(i)+Z_{1}(i), \\
Y(i) & =h_{31} X_{1}(i)+h_{32} X_{2}(i)+Z(i),
\end{aligned}
$$

where $Y_{1}(i)$ and $Y(i)$ are the received signals at the relay and the destination, respectively. 


\section{B. Half-Duplex Mode}

In practice, it is technically challenging to implement the relay in the full-duplex mode under which the relay transmits and receives signals simultaneously within the same frequency band. In contrast, the frequency division or time-division half-duplex mode is more practical [7], [8]. In this paper, we consider the time-division half-duplex mode, where at each time slot the relay listens to the source during the first $\alpha$ fraction of time, and transmits to the destination in the remaining $1-\alpha$ fraction.

Specifically, in the first $\alpha$ portion of the $i$ th time slot, the transmitted signal at the source is denoted by $X_{1}^{(1)}(i)$ with an average power constraint $P_{1}^{(1)}$. The received signals at the relay and the destination are given by:

$$
\begin{aligned}
Y_{1}(i) & =h_{21} X_{1}^{(1)}(i)+Z_{1}(i), \\
Y^{(1)}(i) & =h_{31} X_{1}^{(1)}(i)+Z^{(1)}(i),
\end{aligned}
$$

respectively, where $Z_{1}(i) \sim \mathcal{N}\left(0, N_{1}\right)$ and $Z^{(1)}(i) \sim \mathcal{N}(0, N)$ are correlated AWGN noises with correlation coefficient

$$
\rho_{z}:=\frac{\mathbb{E}\left\{Z_{1} Z^{(1)}\right\}}{\sqrt{N_{1} N}}
$$

In the remaining $1-\alpha$ portion, the transmitted signals at the source and the relay are denoted as $X_{1}^{(2)}(i)$ and $X_{2}(i)$, with average power constraints $P_{1}^{(2)}$ and $P_{2}$, respectively. In this phase, the received signal at the destination is given by:

$$
Y^{(2)}(i)=h_{31} X_{1}^{(2)}(i)+h_{32} X_{2}(i)+Z^{(2)}(i)
$$

where $Z^{(2)}(i) \sim \mathcal{N}(0, N)$ is the AWGN noise at the destination. With memoryless assumption, we have $Z_{1}(i)$ and $Z^{(2)}(i)$ independent of each other.

\section{CAPACity UpPer Bounds AND Achievable Rates}

In this section, we provide the upper and lower bounds on the capacity for both full-duplex and halfduplex Gaussian relay channels with correlated noises. Specifically, max-flow min-cut capacity upper bound is investigated and various relay strategies (DF, CF and AF) are extended to the respective channel settings to obtain the corresponding achievable rates. In addition, we characterize the exact capacity of two special cases, one being achieved by DF and the other achieved by direct transmission (or a special case of CF). These two cases correspond to the Gaussian degraded relay channel and the reversely-degraded relay channel established in [2], respectively. Furthermore, it is shown that the achievable rates for the CF and AF schemes are monotonically decreasing functions over negative correlation coefficient $\rho_{z}$, which implies that negative noise correlation is always helpful for both the $\mathrm{CF}$ and $\mathrm{AF}$ schemes. At the end of this section, we present an alternative channel model for the correlated-noise channel setting. The alternative relay model has a different 
source-relay link gain and independent noises at the relay and the destination, while it has the same achievable rate as the original correlated-noise model, when $\mathrm{CF}$ or $\mathrm{AF}$ scheme is applied.

Note that in this section, all the results are based on a fixed power assignment: $\left\{P_{1}, P_{2}\right\}$ under the fullduplex mode or $\left\{P_{1}^{(1)}, P_{1}^{(2)}, P_{2}\right\}$ under the half-duplex mode; the case with optimal power allocation will be investigated in Section IV For convenience, we define the following function:

$$
\Gamma(x)=\frac{1}{2} \log _{2}(1+x) .
$$

Also denote the normalized channel gains in different links as:

$$
\gamma_{21}=\frac{h_{21}^{2}}{N_{1}}, \quad \gamma_{32}=\frac{h_{32}^{2}}{N}, \quad \gamma_{31}=\frac{h_{31}^{2}}{N} .
$$

\section{A. Max-Flow Min-Cut Upper Bound}

1) Full-Duplex Mode: By applying the max-flow min-cut theorem [2, Theorem 4], we derive an upper bound for the proposed channel model under the full-duplex mode as follows.

Proposition 1 (Upper bound with full-duplex). For the full-duplex Gaussian relay channel with noise correlation defined in Section II-A an upper bound on the capacity is given by:

$$
C_{\text {full }}^{+}=\max _{0 \leq \rho_{x} \leq 1} \min \left\{C_{1-\text { full }}^{+}\left(\rho_{x}\right), C_{2-\text { full }}^{+}\left(\rho_{x}\right)\right\},
$$

where,

$$
\begin{aligned}
& C_{1-\text { full }}^{+}\left(\rho_{x}\right)=\Gamma\left(\gamma_{31} P_{1}+\gamma_{32} P_{2}+2 \rho_{x} \sqrt{\gamma_{31} P_{1} \gamma_{32} P_{2}}\right) \\
& C_{2-\text { full }}^{+}\left(\rho_{x}\right)=\Gamma\left(\frac{P_{1}\left(1-\rho_{x}^{2}\right)\left(\gamma_{21}+\gamma_{31}-2 \rho_{z} \sqrt{\gamma_{21} \gamma_{31}}\right)}{\left(1-\rho_{z}^{2}\right)}\right) .
\end{aligned}
$$

The proof is a trivial extension of that in [2, Theorem 4], which is skipped here.

2) Half-Duplex Mode: As an extension of the max-flow min-cut upper bound in [8, Proposition 1], we have the following upper bound for the half-duplex mode in the correlated-noise case.

Proposition 2 (Upper bound with half-duplex). For the time-division half-duplex Gaussian relay channel with noise correlation defined in Section $[I-B$ assuming a fixed time-division parameter $\alpha$, an upper bound on the channel capacity is given by:

$$
C_{\text {half }}^{+}=\max _{0 \leq \rho_{x} \leq 1} \min \left\{C_{1-\text { half }}^{+}\left(\rho_{x}\right), C_{2-\text { half }}^{+}\left(\rho_{x}\right)\right\},
$$

where

$$
\begin{aligned}
& C_{1-\text { half }}^{+}\left(\rho_{x}\right)=\alpha \Gamma\left(\gamma_{31} P_{1}^{(1)}\right)+(1-\alpha) \Gamma\left(\gamma_{31} P_{1}^{(2)}+\gamma_{32} P_{2}+2 \rho_{x} \sqrt{\gamma_{31} P_{1}^{(2)} \gamma_{32} P_{2}}\right) \\
& C_{2-\text { half }}^{+}\left(\rho_{x}\right)=\alpha \Gamma\left(\frac{\left(\gamma_{21}+\gamma_{31}-2 \rho_{z} \sqrt{\gamma_{21} \gamma_{31}}\right) P_{1}^{(1)}}{1-\rho_{z}^{2}}\right)+(1-\alpha) \Gamma\left(\left(1-\rho_{x}^{2}\right) \gamma_{31} P_{1}^{(2)}\right) .
\end{aligned}
$$


The proof is a trivial extension of that in [8, Proposition 1], which is skipped here.

We next study the achievable rates of different coding strategies, including DF, CF, and AF under both full-duplex and half-duplex modes.

\section{B. Decode-and-Forward}

1) Full-Duplex Mode: In the DF scheme, the relay decodes the message from the source and re-transmits it to the destination. In fact, the message is sent to the destination node through cooperation, which is enabled by the common information at the source and relay nodes. The following rate is achievable with the DF scheme [2]:

$$
R_{\mathrm{df}}=\max _{p\left(x_{1}, x_{2}\right)} \min \left\{I\left(X_{1} ; Y_{1} \mid X_{2}\right), I\left(X_{1}, X_{2} ; Y\right)\right\} .
$$

Here we evaluate the above rate under the full-duplex channel setting given in Section $\amalg-\mathrm{A}$ as follows.

Proposition 3 (Achievable rate for DF with full-duplex). For the full-duplex Gaussian relay channel with noise correlation defined in Section [I-A the achievable rate for the decode-and-forward strategy is given by:

$$
R_{d f-f u l l}=\max _{0 \leq \rho_{x} \leq 1} \min \left\{R_{1-f u l l}\left(\rho_{x}\right), R_{2-\text { full }}\left(\rho_{x}\right)\right\},
$$

where,

$$
\begin{aligned}
& R_{1-\text { full }}\left(\rho_{x}\right)=\Gamma\left(\gamma_{21} P_{1}\left(1-\rho_{x}^{2}\right)\right) \\
& R_{2-\text { full }}\left(\rho_{x}\right)=\Gamma\left(\gamma_{31} P_{1}+\gamma_{32} P_{2}+2 \rho_{x} \sqrt{\gamma_{31} P_{1} \gamma_{32} P_{2}}\right) .
\end{aligned}
$$

2) Half-Duplex Mode: With half-duplex operation, the message is also sent to the destination through cooperation between the source and the relay. However, the relay only decodes the received signal from the source in the first phase of each time slot. In the second phase, the relay re-encodes the received message, and then transmits it to the destination in cooperation with the source.

The achievable rate for DF under half-duplex mode is given by the following proposition.

Proposition 4 (Achievable rate for DF with half-duplex). For the time-division half-duplex Gaussian relay channel with noise correlation defined in Section $[I-B$ assuming a fixed time-division parameter $\alpha$, the achievable rate for the decode-and-forward strategy is given by:

$$
R_{d f-h a l f}=\max _{0 \leq \rho_{x} \leq 1} \min \left\{R_{1-\text { half }}\left(\rho_{x}\right), R_{2-\text { half }}\left(\rho_{x}\right)\right\}
$$

where

$$
\begin{aligned}
& R_{1-\text { half }}\left(\rho_{x}\right)=\alpha \Gamma\left(\gamma_{21} P_{1}^{(1)}\right)+(1-\alpha) \Gamma\left(\left(1-\rho_{x}^{2}\right) \gamma_{31} P_{1}^{(2)}\right) \\
& R_{2 \text {-half }}\left(\rho_{x}\right)=\alpha \Gamma\left(\gamma_{31} P_{1}^{(1)}\right)+(1-\alpha) \Gamma\left(\gamma_{31} P_{1}^{(2)}+\gamma_{32} P_{2}+2 \rho_{x} \sqrt{\gamma_{31} P_{1}^{(2)} \gamma_{32} P_{2}}\right) .
\end{aligned}
$$

The proof for Proposition 4 can be found in [8, Propostion 2]. 
Remark 1. We observe that the DF achievable rates under both full-duplex and half-duplex modes are independent of $\rho_{z}$, and they coincide with the rates in the uncorrelated-noise case. The reason is that the relay completely disregards the noise correlation by decoding the received signal under both full-duplex and half-duplex modes.

3) Capacity-Achieving Case: Based on the upper bound and the achievable rates for the DF scheme, here we present the capacity-achieving case for DF under both full-duplex and half-duplex modes. We show that the capacity-achieving case for DF is corresponding to the Gaussian degraded relay channel in [2].

Theorem 1 (Capacity-achieving case for DF). If the noise correlation coefficient $\rho_{z}$ is equal to $\sqrt{\gamma_{31} / \gamma_{21}}$, the capacity of the relay channel defined in Section $\square$ is achieved by the DF strategy under both full-duplex and half-duplex modes. Such a relay channel is equivalent to the Gaussian degraded relay channel defined in [2], and the capacity under full-duplex mode is given by:

$$
C_{1-f u l l}=\max _{0 \leq \rho_{x} \leq 1} \min \left\{C_{11-f u l l}\left(\rho_{x}\right), C_{12-\text { full }}\left(\rho_{x}\right)\right\} .
$$

where

$$
\begin{aligned}
& C_{11-\text { full }}\left(\rho_{x}\right)=\Gamma\left(\gamma_{21} P_{1}\left(1-\rho_{x}^{2}\right)\right) ; \\
& C_{12 \text {-full }}\left(\rho_{x}\right)=\Gamma\left(\gamma_{31} P_{1}+\gamma_{32} P_{2}+2 \rho_{x} \sqrt{\gamma_{31} P_{1} \gamma_{32} P_{2}}\right) .
\end{aligned}
$$

The capacity under half-duplex mode is given by:

$$
C_{1-\text { half }}=\max _{0 \leq \rho_{x} \leq 1} \min \left\{C_{11-\text { half }}\left(\rho_{x}\right), C_{12-\text { half }}\left(\rho_{x}\right)\right\}
$$

where

$$
\begin{aligned}
& C_{11 \text {-half }}\left(\rho_{x}\right)=\alpha \Gamma\left(\gamma_{21} P_{1}\right)+(1-\alpha) \Gamma\left(\left(1-\rho_{x}^{2}\right) \gamma_{31} P_{1}^{(2)}\right) \\
& C_{12 \text {-half }}\left(\rho_{x}\right)=\alpha \Gamma\left(\gamma_{31} P_{1}^{(1)}\right)+(1-\alpha) \Gamma\left(\gamma_{31} P_{1}^{(2)}+\gamma_{32} P_{2}+2 \rho_{x} \sqrt{\gamma_{31} P_{1}^{(2)} \gamma_{32} P_{2}}\right) .
\end{aligned}
$$

Proof: For the full-duplex mode, we observe that the achievable rate of DF meets the capacity upper bound by substituting $\rho_{z}=\sqrt{\gamma_{31} / \gamma_{21}}$ into (1) and (3).

Now we show that this capacity-achieving case is actually equivalent to the Gaussian degraded relay channel. In [2], the Gaussian degraded relay channel is defined as:

$$
\begin{aligned}
& Y_{1}=X_{1}+Z_{1}, \\
& Y=X_{1}+X_{2}+Z,
\end{aligned}
$$

where $Z=Z_{1}+Z_{2}, Z_{1}$ and $Z_{2}$ are both zero-mean Gaussian noises and independent of each other.

In our model, after normalizing over respective channel gains, the channel can be described as:

$$
\begin{aligned}
\frac{Y_{1}}{h_{21}} & =X_{1}+\frac{Z_{1}}{h_{21}}, \\
\frac{Y}{h_{31}} & =X_{1}+\frac{h_{32}}{h_{31}} X_{2}+\frac{Z}{h_{31}} .
\end{aligned}
$$


For the relay channel to be degraded, the source-relay link is better than the source-destination link, and $\frac{Z}{h_{31}}=\frac{Z_{1}}{h_{21}}+Z_{2}$, where $Z_{1}$ and $Z_{2}$ are independent of each other. Hence the correlation coefficient between $Z$ and $Z_{1}$ is

$$
\rho_{z}=\frac{\mathbb{E}\left\{Z_{1} Z\right\}}{\sqrt{N_{1} N}}=\frac{h_{31}}{h_{21}} \sqrt{\frac{N_{1}}{N}}=\sqrt{\frac{\gamma_{31}}{\gamma_{21}}} .
$$

The converse can also be established, i.e., given a noise correlation coefficient $\rho_{z}=\sqrt{\gamma_{31} / \gamma_{21}}$, we could always construct a corresponding degraded Gaussian relay channel.

The capacity under half-duplex mode and the channel equivalence to the Gaussian degraded relay channel can be derived in a similar way.

\section{Compress-and-Forward}

1) Full-Duplex Mode: In the CF scheme, the relay compresses the received signal with Wyner-Ziv coding [11], and then forwards the binning index to the destination. The destination first decodes the binning index from the relay, and then decodes the compressed signal by viewing the direct link transmission as the side information. Afterwards, the destination decodes the original message by utilizing both the signal from the direct link and the compressed information provided by the relay. The following achievable rate is established with the CF scheme [2]:

$$
R_{\mathrm{cf}}=\sup _{p(\cdot) \in \mathcal{P}^{*}} I\left(X_{1} ; Y, \hat{Y}_{1} \mid X_{2}\right)
$$

subject to the constraint

$$
I\left(X_{2} ; Y\right) \geq I\left(Y_{1} ; \hat{Y}_{1} \mid X_{2}, Y\right) .
$$

Proposition 5 (Achievable rate for CF with full-duplex). For the full-duplex Gaussian relay channel with noise correlation defined in Section $[I-A]$ the achievable rate for the compress-and-forward strategy is given by:

$$
R_{c f-f u l l}=\Gamma\left(P_{1}\left(\gamma_{31}+\frac{\left(\rho_{z} \sqrt{\gamma_{31}}-\sqrt{\gamma_{21}}\right)^{2}}{1-\rho_{z}^{2}+N_{w-f u l l} / N_{1}}\right)\right)
$$

where the quantization noise power $N_{w-f u l l}$ is computed as

$$
N_{w-f u l l}=N_{1} \frac{\left(1-\rho_{z}^{2}\right)+\gamma_{31} P_{1}+\gamma_{21} P_{1}-2 \rho_{z} \sqrt{\gamma_{21} \gamma_{31}} P_{1}}{\gamma_{32} P_{2}} .
$$

The proof is similar to that in [2] and skipped here.

2) Half-Duplex Mode: The relay compresses the received signal in the first phase with Wyner-Ziv coding [11], and then transmits it to the destination in the second phase. We have the following proposition that gives the achievable rate for $\mathrm{CF}$ under half-duplex mode. The proposition is an extended version of [2, Theorem 6] and [8, Proposition 3]; so the proof is skipped here. 
Proposition 6 (Achievable rate for CF with half-duplex). For the time-division half-duplex Gaussian relay channel with noise correlation defined in Section [I-B] assuming a fixed time-division parameter $\alpha$, the achievable rate for the compress-and-forward strategy is given by:

$$
R_{c f-h a l f}=\alpha \Gamma\left(P_{1}^{(1)}\left(\gamma_{31}+\frac{\left(\rho_{z} \sqrt{\gamma_{31}}-\sqrt{\gamma_{21}}\right)^{2}}{1-\rho_{z}^{2}+N_{w-h a l f} / N_{1}}\right)\right)+(1-\alpha) \Gamma\left(\gamma_{31} P_{1}^{(2)}\right),
$$

where the quantization noise power $N_{w-h a l f}$ is computed as

$$
N_{w \text {-half }}=N_{1} \frac{\left(1-\rho_{z}^{2}\right)+\gamma_{21} P_{1}^{(1)}+\gamma_{31} P_{1}^{(1)}-2 \rho_{z} \sqrt{\gamma_{21} \gamma_{31}} P_{1}^{(1)}}{\left(1+\gamma_{31} P_{1}^{(1)}\right)\left(\left(1+\frac{\gamma_{32} P_{2}}{1+\gamma_{31} P_{1}^{(2)}}\right)^{\frac{1-\alpha}{\alpha}}-1\right)} .
$$

Remark 2. We see that unlike the DF strategy, the CF achievable rates under both full-duplex and half-duplex modes depend on the noise correlation coefficient $\rho_{z}$, which means that the $\mathrm{CF}$ scheme has the potential to exploit the noise correlation.

Remark 3. It has been shown in some previous works [2], [7] that the direct transmission from the source to the destination can achieve a better rate than the DF strategy if the source-relay link is weaker than the direct source-destination link. The reason is that the DF strategy requires the relay to completely decode the message from the source, which creates a bottleneck on the achievable rate when the link from the source to the relay is relatively weak. However, the CF strategy does not incur such a problem since the relay only compresses the received signal. Moreover, the CF strategy always incorporates the direct transmission as a special case: By setting the compressed signal as a constant, we have the constraint in [2, Theorem 6] always satisfied, and the $\mathrm{CF}$ achievable rate degrades to the capacity of the point-to-point link between the source and the destination. The direct transmission rate under full-duplex mode with the Gaussian channel setting can be easily computed as

$$
R_{\text {direct-full }}=I\left(X_{1} ; Y\right)=\Gamma\left(\gamma_{31} P_{1}\right)
$$

Similarly, under half-duplex mode, the direct link transmission rate can be easily derived as:

$$
R_{\text {direct-half }}=I\left(X_{1}^{(1)} ; Y^{(1)}\right)+I\left(X_{1}^{(2)} ; Y^{(2)}\right)=\alpha \Gamma\left(\gamma_{31} P_{1}^{(1)}\right)+(1-\alpha) \Gamma\left(\gamma_{31} P_{1}^{(2)}\right),
$$

where we have $R_{\text {direct-half }}=\Gamma\left(\gamma_{31} P_{1}\right)=R_{\text {direct-full }}$ if $P_{1}^{(1)}=P_{1}^{(2)}=P_{1}$.

Regarding the achievable rate for the CF scheme under both full-duplex and half-duplex modes, we have the following theorem:

Theorem 2 (Negative correlation always helps CF). For the Gaussian relay channel with noise correlation defined in Section [I] the achievable rates of CF under both full-duplex and half-duplex modes are monotonically decreasing functions over $\rho_{z}$ when $\rho_{z} \in[-1,0]$. Therefore, negative correlation always increases the $C F$ achievable rates compared to the case of independent noises with $\rho_{z}=0$.

Proof: We start with the CF scheme under full-duplex mode. The same conclusion can be drawn for the half-duplex mode in a similar way. 
Since $\Gamma$ is a monotonically increasing function, it suffices to show that its argument in (7) is monotonically decreasing over negative $\rho_{z}$. Denote the second item inside $\Gamma$ of (7) by $G\left(\rho_{z}\right)$ (while the first one is constant over $\left.\rho_{z}\right)$ :

$$
\begin{aligned}
G\left(\rho_{z}\right) & =\frac{P_{1}\left(\rho_{z} \sqrt{\gamma_{31}}-\sqrt{\gamma_{21}}\right)^{2}}{1-\rho_{z}^{2}+N_{w} / N_{1}} \\
& \triangleq \frac{G_{1} \rho_{z}^{2}-G_{2} \rho_{z}+G_{3}}{-G_{4} \rho_{z}^{2}-G_{5} \rho_{z}+G_{6}}, \text { with } G_{i}>0, i=1, \ldots 6, \text { when } \rho_{z} \text { is negative. }
\end{aligned}
$$

The first order derivative of $G\left(\rho_{z}\right)$ over $\rho_{z}$ is

$$
G^{\prime}\left(\rho_{z}\right)=\frac{-\left(G_{1} G_{5}+G_{2} G_{4}\right) \rho_{z}^{2}+2\left(G_{1} G_{6}+G_{3} G_{4}\right) \rho_{z}+G_{3} G_{5}-G_{2} G_{6}}{\left(-G_{4} \rho_{z}^{2}-G_{5} \rho_{z}+G_{6}\right)^{2}}
$$

It can be easily seen that when $\rho_{z}$ is negative, the derivative of $G\left(\rho_{z}\right)$ is negative with $G_{3} G_{5}-G_{2} G_{6}<0$. With the above definition of $G_{i}$ 's, we have that

$$
G_{3} G_{5}-G_{2} G_{6}=\frac{-2\left(1+\gamma_{31} P_{1}+\gamma_{32} P_{2}\right) \sqrt{\gamma_{21} \gamma_{31}} P_{1}}{\gamma_{32} P_{2}}<0 .
$$

Therefore, we claim that the achievable rate for CF under full-duplex mode is strictly decreasing when the noise correlation is negative. In other words, the negative correlation always helps compared with the case of independent noises $\left(\rho_{z}=0\right)$ under full-duplex mode when $\mathrm{CF}$ is applied.

The proof for the case of CF strategy under half-duplex mode is similar and hence omitted here.

Remark 4. Intuitively, the reason for the monotonic decrease in the $\mathrm{CF}$ rate over negative $\rho_{z}$ is the increase in the effective noise power perceived at the destination, when the negative $\rho_{z}$ approaches zero. In other words, the received signal-to-noise-ratio at the destination is monotonically decreasing when the correlation coefficient $\rho_{z}$ is negative, which yields the conclusion in Theorem 2

Remark 5. For the positive noise correlation $\left(\rho_{z}>0\right)$, the achievable rate for the $\mathrm{CF}$ scheme is not a monotonic function any more. To some extent, we could say that compared with the uncorrelated-noise case, the positive noise correlation hurts the $\mathrm{CF}$ scheme for $\rho_{z} \in\left(0, \rho_{z}^{\prime}\right)$, where $\rho_{z}^{\prime}$ satisfies $G\left(\rho_{z}^{\prime}\right)=G(0)$. However, the rate at $\rho_{z}=1$ coincides with the rate at $\rho_{z}=-1$, which means that the positive correlation may also help when $\rho_{z} \in\left[\rho_{z}^{\prime}, 1\right]$. The detailed proof of the above conclusions is given in Appendix $\mathrm{A}$

3) Capacity-Achieving Case: Here we present another capacity result, where the capacity is achieved with either $\mathrm{CF}$ or direct link transmission. In fact, this case is equivalent to the Gaussian reversely-degraded relay channel in [2].

Theorem 3 (Capacity-achieving case for $\mathbf{C F ) . ~ I f ~ t h e ~ n o i s e ~ c o r r e l a t i o n ~ c o e f f i c i e n t ~} \rho_{z}$ is equal to $\sqrt{\gamma_{21} / \gamma_{31}}$, the capacity of the relay channel defined in Section $\square$ is achieved by the direct link transmission from the source to the destination (a special case of CF) under both full-duplex and half-duplex modes. This case is equivalent to the Gaussian reversely-degraded relay channel defined in [2]. The capacity under full-duplex mode is given by:

$$
C_{2-\text { full }}=\Gamma\left(\gamma_{31} P_{1}\right),
$$


and the capacity under half-duplex mode is given by:

$$
C_{2-\text { half }}=\alpha \Gamma\left(\gamma_{31} P_{1}^{(1)}\right)+(1-\alpha) \Gamma\left(\gamma_{31} P_{1}^{(2)}\right) .
$$

Proof: The achievability can be shown by setting the compressed signal in the relay CF operation as a constant, which means that the rate of CF strategy in this case is actually the direct link transmission rate under both full-duplex and half-duplex modes.

For the converse part, the upper bound $C_{\text {full }}^{+}$under full-duplex mode in (11) is further bounded by:

$$
\begin{aligned}
C_{\text {full }}^{+} & \leq \max _{0 \leq \rho_{x} \leq 1} C_{2 \text { full }}^{+} \\
& =\Gamma\left(\frac{\left(1-\rho_{x}^{2}\right) P_{1}\left(\gamma_{21}+\gamma_{31}-2 \rho_{z} \sqrt{\gamma_{21} \gamma_{31}}\right)}{\left(1-\rho_{z}^{2}\right)}\right) \\
& =\Gamma\left(\frac{P_{1}\left(\gamma_{21}+\gamma_{31}-2 \rho_{z} \sqrt{\gamma_{21} \gamma_{31}}\right)}{\left(1-\rho_{z}^{2}\right)}\right) .
\end{aligned}
$$

By substituting $\rho_{z}=\sqrt{\gamma_{21} / \gamma_{31}}$ into $(15)$, we have

$$
C_{\text {full }}^{+} \leq \Gamma\left(\gamma_{31} P_{1}\right)
$$

which establishes the converse part under the full-duplex mode.

The converse part under half-duplex mode can be proved by substituting $\rho_{z}=\sqrt{\gamma_{21} / \gamma_{31}}$ into (2):

$$
\begin{aligned}
C_{\text {half }}^{+} & \leq \max _{0 \leq \rho_{x} \leq 1} C_{2 \text {-half }}^{+}\left(\rho_{x}\right) \\
& =\max _{0 \leq \rho_{x} \leq 1}\left(\alpha \Gamma\left(\gamma_{31} P_{1}^{(1)}\right)+(1-\alpha) \Gamma\left(\left(1-\rho_{x}^{2}\right) \gamma_{31} P_{1}^{(2)}\right)\right) \\
& =\alpha \Gamma\left(\gamma_{31} P_{1}^{(1)}\right)+(1-\alpha) \Gamma\left(\gamma_{31} P_{1}^{(2)}\right) .
\end{aligned}
$$

By combining both the achievability and converse parts, we see that when $\rho_{z}=\sqrt{\gamma_{21} / \gamma_{31}}$, the capacity is achieved by the special CF scheme: the direct link transmission.

The capacity-achieving case here is actually equivalent to the reversely-degraded Gaussian relay channel defined in [2], which is described as:

$$
\begin{aligned}
& Y_{1}=X_{1}+Z_{1}, \\
& Y=X_{1}+X_{2}+Z,
\end{aligned}
$$

where $Z_{1}=Z+Z_{2}, Z$ and $Z_{2}$ are both zero-mean Gaussian noises and independent of each other.

In our model, the channel can be described as:

$$
\begin{aligned}
\frac{Y_{1}}{h_{21}} & =X_{1}+\frac{Z_{1}}{h_{21}}, \\
\frac{Y}{h_{31}} & =X_{1}+\frac{h_{32}}{h_{31}} X_{2}+\frac{Z}{h_{31}} .
\end{aligned}
$$


For the relay channel to be reversely-degraded, the source-destination link is better than the source-relay link, and $\frac{Z_{1}}{h_{21}}=\frac{Z}{h_{31}}+Z_{2}$, where $Z$ and $Z_{2}$ are independent of each other. Therefore, the correlation coefficient between $Z$ and $Z_{1}$ is

$$
\rho_{z}=\frac{\mathbb{E}\left\{Z_{1} Z\right\}}{\sqrt{N_{1} N}}=\frac{h_{21}}{h_{31}} \sqrt{\frac{N}{N_{1}}}=\sqrt{\frac{\gamma_{21}}{\gamma_{31}}} .
$$

The converse can also be established, i.e., we could always construct a corresponding reversely-degraded Gaussian relay channel given a noise correlation coefficient $\rho_{z}=\sqrt{\gamma_{21} / \gamma_{31}}$. The capacity under half-duplex mode and the channel equivalence to a reversely-degraded Gaussian relay channel can be derived in a similar way.

\section{Amplify-and-Forward}

In the AF scheme (under half-duplex mode), the source transmits the information to the relay and the destination in the first phase, and keeps silent in the second phase. The relay forwards a scaled version of the received signal to the destination without decoding or compressing. The destination decodes the information at the end of each time slot by using an Maximum Ratio Combining (MRC) decoder. The time-division parameter $\alpha$ is fixed to 0.5 since the length of the codewords in both phases must be the same.

The achievable rate for $\mathrm{AF}$ is given by the following proposition, for which [9. Eq.(12)] is a special case with independent noises.

Proposition 7 (Achievable rate for AF). For the time-division half-duplex Gaussian relay channel with noise correlation defined in Section $[I-B$ the achievable rate for the amplify-and-forward strategy is given by:

$$
R_{a f}=\frac{1}{2} \Gamma\left(\gamma_{31} P_{1}^{(1)}+\frac{\gamma_{32} P_{2}\left(\rho_{z} \sqrt{\gamma_{31}}-\sqrt{\gamma_{21}}\right)^{2} P_{1}^{(1)}}{1+\gamma_{21} P_{1}^{(1)}+\gamma_{32} P_{2}\left(1-\rho_{z}^{2}\right)}\right) .
$$

Regarding the AF achievable rate, we have a similar observation to that of the CF scheme, which is stated as follows:

Theorem 4 (Negative correlation always helps AF). For the time-division half-duplex Gaussian relay channel with noise correlation defined in Section $[I-B$ the achievable rate of $A F$ is a monotonically decreasing function over $\rho_{z}$ when $\rho_{z} \in[-1,0]$, which means that negative correlation always increases the AF achievable rate compared to the case of uncorrelated noises with $\rho_{z}=0$.

Proof: By taking the derivative of the item inside the $\Gamma$ function in (16) over $\rho_{z}$, it can be shown that the first order derivative is always negative when $\rho_{z}$ is negative (using the similar proof as in Theorem 2). Combined with the monotonicity of the $\Gamma$ function, we conclude that $R_{\mathrm{af}}$ is strictly decreasing when $\rho_{z}$ is between -1 and 0 .

Remark 6. In fact, the intuitive reasoning of Theorem 4 is given as follows. When the signals from the source and the relay add up together via MRC, the noise power decreases if the noise correlation coefficient is 
negative. Therefore, the signal-to-noise ratio at the destination is increased, which leads to the improvement of the achievable rate compared with the uncorrelated-noise case.

\section{E. Alternative Relay Model for Compress-and-Forward and Amplify-and-Forward}

From the achievable rates of the $\mathrm{CF}$ and $\mathrm{AF}$ schemes shown in (7), (8), and (16), we observe that they can be achieved by another alternative relay channel with independent noises and an appropriately defined source-relay link gain, which is shown in Fig. 2. In particular, the power of the AWGN noise $Z_{1}^{\prime}$ at the relay

is $\left(1-\rho_{z}^{2}\right) N_{1}$ and the source-relay channel gain is $h_{21}^{\prime}=\left|h_{21}-h_{31} \rho_{z} \sqrt{\frac{N_{1}}{N}}\right|$, with other parameters remaining the same. Under both the full-duplex and half-duplex modes, the achievable rates of the CF and AF schemes in the alternative relay model are the same as those for the correlated-noise model discussed previously.

When $\rho_{z}=1$ or $\rho_{z}=-1$, the relay becomes noiseless in the alternative relay model and the CF achievable rate is $\Gamma\left(\gamma_{31} P_{1}+\gamma_{32} P_{2}\right)$ under full-duplex mode. However, for the AF strategy, the achievable rate at $\rho_{z}=1$ is smaller than the rate at $\rho_{z}=-1$ since the source-relay link still affects the achievable rate even with a noiseless relay.

Regarding the special case in Proposition 3, we find that in the corresponding alternative relay model, the source-relay channel gain becomes 0 when $\rho_{z}=\sqrt{\gamma_{21} / \gamma_{31}}$, such that the compress-and-forward scheme degrades to the direct link transmission. Moreover, the conclusions in Theorems 2 and 4 can be equivalently shown with the alternative relay model, since the normalized channel gain between the source and the relay is a strictly decreasing function over negative $\rho_{z}$.

Such an alternative relay model with independent noises for CF and AF is especially useful when we derive the optimal power allocation policy for the respective strategies, since we could extend the results for the traditional relay channel with independent noises, which will be discussed in the next section.

\section{Optimal Power Allocation}

In this section, we investigate the optimal power allocation at the source and the relay to maximize the achievable rates of $\mathrm{CF}$ and $\mathrm{AF}$ under the correlated-noise channel settings. Two different types of power constraints are considered: 1) The relay has an individual power constraint $P_{2 \_} t$ with the source power being fixed as $\left.P_{1} ; 2\right)$ The total power across the source and the relay is constrained as $P_{t}$. With the individual power constraint at the relay, we have the conclusion that the relay should always utilize all the power budget $P_{2_{-} t}$ to maximize the achievable rate instead of using only part of it at an arbitrary noise correlation coefficient $\rho_{z}$. With the total power constraint across the source and the relay, we observe that the achievable rates for the $\mathrm{CF}$ scheme under full-duplex mode and the AF scheme are concave functions over the power allocation factor. Hence, there exists a unique optimal point and the closed-form results are provided for the optimal power allocation. However, with the total power constraint, the power assignment for the CF strategy under 
half-duplex mode is not considered here since no closed-form can be derived [8]. We also skip the optimal power allocation for the DF scheme, which is independent of the noise correlation and can be referred to [8] for more details.

\section{A. Individual Power Constraint at the Relay}

With an individual power constraint at the relay, we maximize the achievable rates for $\mathrm{CF}$ and $\mathrm{AF}$. When the noises at the relay and the destination are correlated, it is not clear whether the relay should forward the signal plus noise with the maximum power. We address this problem as follows.

The optimization problem can be formulated as:

$$
\begin{array}{cl}
\max _{P_{2}} & R_{\text {cf-full }} \quad\left(\text { or } \quad R_{\text {cf-half }}, \quad R_{\mathrm{af}}\right) \\
\text { s.t. } & P_{2} \leq P_{2 \_},
\end{array}
$$

for the CF scheme under full-duplex, half-duplex, and the AF scheme, respectively.

Theorem 5. For the Gaussian relay channel with noise correlation defined in Section III and an individual power constraint at the relay, the relay should always use all the power budget $P_{2 \_t}$ to achieve higher rates for both $C F$ and AF strategies at any $\rho_{z} \in[-1,1]$.

Proof: With the achievable rates for $\mathrm{CF}$ and $\mathrm{AF}$ in (7), (8), and (16), it is not straightforward to check whether the relay should use all of its power. However, with the alternative relay model defined in Section III-E we can easily show that the relay should use all of its power to maximize the CF or AF achievable rates. We take the $\mathrm{CF}$ achievable rate under full-duplex mode as an example, while the same argument holds for the CF scheme under half-duplex mode and the AF scheme.

When $\rho_{z}=1$ or -1 , the achievable rate for CF under full-duplex mode is $R_{\mathrm{cf} \text {-full }}=\Gamma\left(\gamma_{31} P_{1}+\gamma_{32} P_{2}\right)$, which is an monotonically increasing function over the variable $P_{2}$. Therefore, the relay should use all the power budget $P_{2_{-} t}$ if $\rho_{z}=1$ or -1 .

If the noises are not fully-correlated, i.e., $\rho_{z} \in(-1,1)$, we adopt the achievable rate under the alternative relay model defined in Section $\amalg$ II-E

$$
R_{\mathrm{cf}-\text { full }}=\Gamma\left(\gamma_{31} P_{1}+\frac{\gamma_{21}^{\prime} P_{1} \gamma_{32} P_{2}}{1+\gamma_{21}^{\prime} P_{1}+\gamma_{31} P_{1}+\gamma_{32} P_{2}}\right),
$$

where $\gamma_{21}^{\prime}$ is defined as the effective normalized channel gain under the alternative relay model:

$$
\gamma_{21}^{\prime}=\frac{\left(\sqrt{\gamma_{21}}-\rho_{z} \sqrt{\gamma_{31}}\right)^{2}}{\left(1-\rho_{z}^{2}\right)} .
$$

The above achievable rate for CF under full-duplex mode is also an increasing function over the variable $P_{2}$ for $\rho_{z} \in(-1,1)$. The same conclusion can be drawn for the $\mathrm{CF}$ rate under half-duplex mode and the AF rate, in similar ways. Therefore, we conclude that the relay should use all of its power to maximize the achievable rates under the correlated-noise relay channel setting. 


\section{B. Total Power Constraint}

1) Compress-and-Forward under Full-Duplex: The source transmits its codeword with power $P_{1}$, and the relay maps the compressed signal to a new codeword with power $P_{2}$. The total power is constrained to $P_{t}$, i.e., $P_{1}+P_{2} \leq P_{t}$, such that the optimization problem can be formulated as:

$$
\begin{aligned}
\max _{P_{1}, P_{2}} & R_{\text {cf-full }} \\
\text { s.t. } & P_{1}+P_{2} \leq P_{t},
\end{aligned}
$$

where $R_{\text {cf-full }}$ is the achievable rate of CF under full-duplex mode given in (7). Since $\Gamma$ function is monotonically increasing, we can maximize the item inside the $\Gamma$ function instead of $R_{\text {cf-full }}$ itself. The optimal power allocation policy is provided in the following theorem.

Theorem 6. With a total power constraint across the source and the relay, the achievable rate for the CF scheme under full-duplex mode is a concave function over the power allocation parameters $P_{1}$ and $P_{2}$. To maximize the achievable rate in (7) for the CF scheme under full-duplex mode, if the noises are fully-correlated $\left(\rho_{z}=1\right.$ or -1$)$, the optimal power allocation policy is :

$$
\begin{aligned}
& P_{1}^{*}=\left\{\begin{array}{cc}
\epsilon & \text { if } \gamma_{31}<\gamma_{32}, \epsilon \text { is an arbitrarily small positive number } \\
P_{t} & \text { otherwise, }
\end{array}\right. \\
& P_{2}^{*}=P_{t}-P_{1}^{*} .
\end{aligned}
$$

If the noises are not fully-correlated with $\rho_{z} \in(-1,1)$, the optimal power allocation is

$$
\begin{aligned}
& P_{1}^{*}= \begin{cases}\frac{1}{\gamma_{32}-\gamma_{21}^{\prime}-\gamma_{31}}\left(1+\gamma_{32} P_{t}-\sqrt{\frac{\gamma_{32} \gamma_{21}^{\prime}\left(1+\gamma_{32} P_{t}\right)\left(1+\gamma_{31} P_{t}+\gamma_{21}^{\prime} P_{t}\right)}{\left(\gamma_{32}-\gamma_{31}\right)\left(\gamma_{21}^{\prime}+\gamma_{31}\right)}}\right) & \text { if } \gamma_{21}^{\prime}\left(\gamma_{32}-\gamma_{31}\right) P_{t}>\gamma_{31}\left(1+\gamma_{31} P_{t}\right) \\
P_{t} & \text { otherwise, }\end{cases} \\
& P_{2}^{*}=P_{t}-P_{1}^{*},
\end{aligned}
$$

where $\gamma_{21}^{\prime}$ is the normalized effective source-relay gain in the alternative relay model defined in (17).

The detailed proof is given in Appendix $\mathrm{B}$ with the outline sketched as: If the noises are fully-correlated, the achievable rate of $\mathrm{CF}$ under full-duplex mode is $\Gamma\left(\gamma_{31} P_{1}+\gamma_{32} P_{2}\right)$. If $\gamma_{31}<\gamma_{32}$, the source power should be as small as possible, and correspondingly, the relay power should be as large as possible due to the total power constraint. Otherwise, when $\gamma_{31} \geq \gamma_{32}$, the source node should use all the power $P_{t}$ and the relay keeps silent to maximize the achievable rate. If the noises are not fully-correlated, first we claim that the constraint is active at the optimal point: For any given power $P_{1}$ at the source, the relay should always utilize all the available remaining power to obtain higher rate according to Theorem [5. Therefore, we can substitute $P_{2}$ by $P_{t}-P_{1}$ in the objective function. By taking the second-order derivative of the objective function over $P_{1}$ under the alternative relay model mentioned in Section [II-E, we see that the second-order derivative is always negative, which implies that the objective function is concave over the variable $P_{1}$. Then the optimal solution can be solved by setting the first-order derivative to zero, which results in a second-order polynomial equation with 
respect to $P_{1}$. Due to the properties of the derived second-order polynomial equation, either only one of the roots is in the range $\left(0, P_{t}\right)$, or the boundary point $P_{1}=P_{t}$ is the optimal point, which is $P_{1}^{*}$ in (20). Hence, the power allocated to the relay is $P_{2}^{*}=P_{t}-P_{1}^{*}$, which is given in 20).

2) Amplify-and-Forward: The source transmits the message in the first phase and keeps silent in the second phase. The relay forwards the amplified signal to the destination in the second phase. Due to the fact that both the source and the relay only use half of the slot for transmission, the effective average power levels at the source and the relay are $P_{1}^{(1)} / 2$ and $P_{2} / 2$, respectively. As a result, the total power constraint can be modified as $P_{1}^{(1)}+P_{2} \leq 2 P_{t}$. Accordingly, the power allocation optimization problem can be cast as:

$$
\begin{aligned}
\underset{P_{1}^{(1)}, P_{2}}{\max } & R_{\mathrm{af}} \\
\text { s.t. } & P_{1}^{(1)}+P_{2} \leq 2 P_{t},
\end{aligned}
$$

where $R_{\mathrm{af}}$ is the achievable rate for the AF scheme given in (16).

Similar to the power allocation problem for $\mathrm{CF}$ under full-duplex mode, we consider the item inside the $\Gamma$ function of $R_{\mathrm{af}}$ as the objective function, which is also concave over $P_{1}^{(1)}$ when applying the alternative relay model defined in Section

Theorem 7. With a total power constraint over the source and the relay, the achievable rate for the AF scheme is a concave function over the power allocation parameters $P_{1}^{(1)}$ and $P_{2}$. To maximize the achievable rate $R_{a f}$ for the AF scheme in (16), if the noises are positively fully-correlated $\left(\rho_{z}=1\right)$, the optimal power allocation policy is:

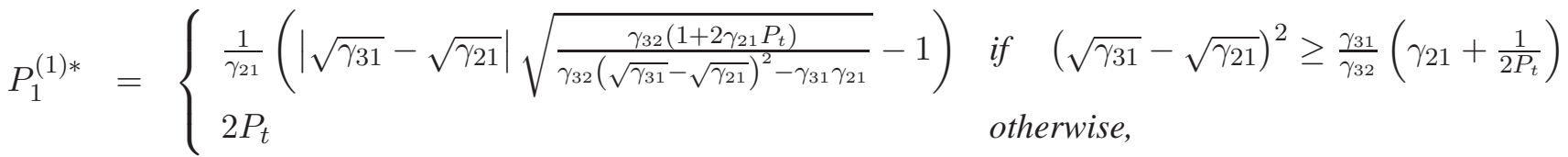

$$
\begin{aligned}
& P_{2}^{*}=2 P_{t}-P_{1}^{(1) *} \text {. }
\end{aligned}
$$

If the noises are negatively fully-correlated $\left(\rho_{z}=-1\right)$, the optimal power allocation policy is:

$$
\begin{aligned}
P_{1}^{(1) *} & = \begin{cases}\frac{1}{\gamma_{21}}\left(\left(\sqrt{\gamma_{31}}+\sqrt{\gamma_{21}}\right) \sqrt{\left.\frac{\gamma_{32}\left(1+2 \gamma_{21} P_{t}\right)}{\gamma_{32}\left(\sqrt{\gamma_{31}}+\sqrt{\gamma_{21}}\right)^{2}-\gamma_{31} \gamma_{21}}-1\right)}\right. & \text { if }\left(\sqrt{\gamma_{31}}+\sqrt{\gamma_{21}}\right)^{2} \geq \frac{\gamma_{31}}{\gamma_{32}}\left(\gamma_{21}+\frac{1}{2 P_{t}}\right) \\
2 P_{t} & \text { otherwise, }\end{cases} \\
P_{2}^{*}=2 P_{t}-P_{1}^{(1) *} . &
\end{aligned}
$$

If the noises are not fully-correlated with $\rho_{z} \in(-1,1)$, the optimal power allocation is

$$
\begin{aligned}
P_{1}^{(1) *} & = \begin{cases}\frac{1}{\gamma_{32}-\gamma_{21}^{\prime}}\left(1+2 \gamma_{32} P_{t}-\sqrt{\left.\frac{\gamma_{32} \gamma_{21}^{\prime}\left(1+2 \gamma_{32} P_{t}\right)\left(1+2 \gamma_{21}^{\prime} P_{t}\right)}{\gamma_{32} \gamma_{21}^{\prime}-\gamma_{31} \gamma_{21}^{\prime}+\gamma_{31} \gamma_{32}}\right)}\right. & \text { if } \gamma_{21}^{\prime} \geq \frac{\gamma_{31}}{\gamma_{32}}\left(\gamma_{21}+\frac{1}{2 P_{t}}\right) \\
2 P_{t} & \text { otherwise, }\end{cases} \\
P_{2}^{*} & =2 P_{t}-P_{1}^{(1) *} .
\end{aligned}
$$

where $\gamma_{21}^{\prime}$ is the normalized effective source-relay gain in the alternative relay model defined in (17).

The proof is similar to that of Theorem 6, and hence skipped here. 


\section{NUMERICAL RESULTS}

In this section, we compare the achievable rates of $\mathrm{DF}, \mathrm{CF}$, and $\mathrm{AF}$ against the capacity upper bound under both full-duplex and half-duplex modes. For the full duplex mode, first we consider the fixed power allocation scenario and set both $P_{1}$ (average power at the source) and $P_{2}$ (average power at the relay) to 1 . We also illustrate the performance of the CF scheme using optimal power allocation. For the half-duplex mode, we first set $\alpha=0.5$, and compare the achievable rates against the capacity upper bound under fixed power assignment. Then we present the numerical results after optimizing the time-division parameter $\alpha$ under half-duplex mode. Finally we show the improvement due to the optimal power allocation for the AF scheme. In both full-duplex and half-duplex modes, we set $N=N_{1}=1$.

We will apply the following channel model for all the numerical comparisons: the source, relay, and destination are aligned on a line. The distance between the source and the relay is $d(0<d<1)$, and the distance between the source and the destination is 1 . The channel amplitude is inversely proportional to the distance, which means:

$$
h_{21}=\frac{1}{d}, h_{32}=\frac{1}{1-d}, h_{31}=1, \quad d \in(0,1)
$$

\section{A. Full-Duplex Mode}

1) Fixed Power Allocation: For the fixed power allocation $\left\{P_{1}, P_{2}\right\}=\{1,1\}$, we first evaluate the upper bound and the achievable rates for DF, CF, and direct link transmission, versus the noise correlation coefficient $\rho_{z}$. The cases for different channel parameters $(d=0.4$ and $d=0.8)$ are shown in Fig. 3(a) and Fig. 3(b), respectively.

From Fig. 3(a) and Fig. 3(b), we see that when the relay is close to the source $(d=0.4)$, DF always performs the best, which means that the exploitation of noise correlation is not necessary since the received SNR at the relay is high enough. However, when the relay is close to the destination $(d=0.8)$, CF outperforms DF for most of $\rho_{z} \in[-1,1]$, since the SNR at the relay is relatively low such that the exploitation of noise correlation brings more benefits. Furthermore, we observe that the DF rate achieves the capacity upper bound when $\rho_{z}=\sqrt{\gamma_{31} / \gamma_{21}}$, which confirms Theorem 1 Meanwhile, Theorem 2 is also verified in these figures, i.e., the achievable rate for CF under full-duplex mode is strictly decreasing over negative $\rho_{z}$.

2) Optimal Power Allocation for CF: The comparison between the achievable rates for CF under fixed power allocation and optimal power allocation is shown in Fig. 4(a), which shows that the optimal power allocation can bring more profit near fully-correlated points if the relay is close to the source $(d=0.4)$. If the relay is close to the destination $(d=0.8)$, the improvement is more apparent when the channel is more like a degraded relay channel (near $\rho_{z}=\sqrt{\gamma_{31} / \gamma_{21}}=0.8$ ).

The optimal power allocated to the source are shown in Fig. 4(b) for $d=0.4$ and $d=0.8$, respectively. We see that the source should use more power if the channel behaves more like degraded, i.e., near $\rho_{z}=0.4$ when 
$d=0.4$, and $\rho_{z}=0.8$ when $d=0.8$, respectively. Moreover, with the same correlation coefficient $\rho_{z}$, if the relay is close to the destination $(d=0.8)$, the source should use more power than the case in which the relay is close to the source $(d=0.4)$.

\section{B. Half-Duplex Mode}

1) Fixed $\alpha$ : First, the time-division parameter $\alpha$ is set to 0.5 in the DF and CF schemes for a fair comparison with $\mathrm{AF}$ that has a requirement of $\alpha=0.5$. The power assignments are $\left\{P_{1}^{(1)}, P_{1}^{(2)}, P_{2}\right\}=\{1,1,2\}$ for DF and $\mathrm{CF}$, but $\left\{P_{1}^{(1)}, P_{1}^{(2)}, P_{2}\right\}=\{2,0,2\}$ for the $\mathrm{AF}$ scheme.

The upper bound, the $\mathrm{CF}$ rate, the $\mathrm{DF}$ rate, the $\mathrm{AF}$ rate, and the achievable rate of direct transmission are plotted in Fig. 5(a) and Fig. 5(b) for different $d$ values. The analysis of this scenario is similar to the full-duplex case in Section $\mathrm{V}-\mathrm{A}$ In particular, when the relay is close to the destination, the AF scheme can even beat $\mathrm{DF}$ and is close to the upper bound for all negative $\rho_{z}$. However, when the noise correlation coefficient $\rho_{z}$ is close to $1, \mathrm{CF}$ is still the best strategy. When $\rho_{z}<0$, Theorems 2 and 4 are verified by showing that negative noise correlation helps CF and AF improve the performance. Moreover, the capacity-achieving case for the DF scheme under half-duplex mode is confirmed as in Theorem 1

2) Optimization over $\alpha$ : We now consider the improved upper bound and achievable rates via optimizing $\alpha$ for DF and CF strategies, with the results shown in Fig. 6(a) and Fig. 6(b) The power allocation is still fixed to $\left\{P_{1}^{(1)}, P_{1}^{(2)}, P_{2}\right\}=\{1,1,2\}$ for both $\mathrm{CF}$ and DF.

Compared to the performance of $\alpha=0.5$ in Fig. 5(a) and Fig. 5(b) we see that the upper bound and the achievable rates for DF and CF are increased by optimizing over $\alpha$. When $d=0.8, \mathrm{CF}$ is not dominant over DF any more for most of $\rho_{z} \in[-1,1]$, which is different from the performance at $\alpha=0.5$. The results in Fig. 6(a) and Fig. 6(b) also show that the capacity-achieving points for DF are still $\rho_{z}=\sqrt{\gamma_{31} / \gamma_{21}}$, which means that Theorem 1 holds at the optimal $\alpha$.

3) Optimal Power Allocation for AF: The performance comparisons between AF with optimal power allocation and AF with fixed power allocation are shown in Fig. 7(a) for different $d$ values. We can draw a similar conclusion as in the case of optimal power allocation for CF under full-duplex mode, except that the optimal power allocation can no longer bring much benefit when the noise correlation coefficient is close to 1 if the relay is close to the source.

The optimal power allocated to the source is shown in Fig. 7(b) Unlike that of CF in Fig. 4(b), the source now obtains more and more power when $\rho_{z}$ goes to 1 . Basically the reason is that the relay signal should use less power to forward less noise if the noises are more positively correlated.

\section{CONCLUSION}

We have obtained upper and lower bounds on the capacity of Gaussian relay channels with correlated noises at the relay and the destination. Specifically, the max-flow min-cut upper bound was first evaluated; then DF, CF, 
and AF strategies were applied to derive the respective achievable rates. We also characterized two capacityachieving cases, which bear the equivalence to the degraded relay channel and the reversely-degraded one established in [2], respectively. It was also shown that the achievable rates of CF and AF are strictly decreasing functions over negative $\rho_{z}$, which means that negative noise correlations always help in CF and AF compared to the uncorrelated case. The optimal power allocation for $\mathrm{CF}$ and AF strategies was also investigated. With an individual power constraint at the relay, the relay should always use all the power to maximize the achievable rates under the correlated-noise channel setting. With a total power constraint over the source and the relay, we proved that the achievable rates for $\mathrm{CF}$ under full-duplex mode and AF are concave functions over the transmission power and closed-form results were given. Numerical examples were provided to compare the performance of various coding strategies.

\section{APPENDIX A}

In this appendix, we prove that the positive correlation may hurt or help the CF achievable rate over two distinct regions. Recall that the first order derivative of $G\left(\rho_{z}\right)$ in (11) is given as

$$
G^{\prime}\left(\rho_{z}\right)=\frac{-\left(G_{1} G_{5}+G_{2} G_{4}\right) \rho_{z}^{2}+2\left(G_{1} G_{6}+G_{3} G_{4}\right) \rho_{z}+G_{3} G_{5}-G_{2} G_{6}}{\left(-G_{4} \rho_{z}^{2}-G_{5} \rho_{z}+G_{6}\right)^{2}},
$$

where the numerator determines the sign of $G^{\prime}\left(\rho_{z}\right)$. When $\rho_{z}=0$, the numerator is reduced to $G_{3} G_{5}-G_{2} G_{6}<$ 0 , which implies that $G^{\prime}(0)<0$. When $\rho_{z}=1$, the first order derivative of $G\left(\rho_{z}\right)$ is

$$
\begin{aligned}
G^{\prime}(1) & =\frac{-G_{1} G_{5}-G_{2} G_{4}+2 G_{1} G_{6}+2 G_{3} G_{4}+G_{3} G_{5}-G_{2} G_{6}}{\left(-G_{4}-G_{5}+G_{6}\right)^{2}} \\
& =\frac{2 P_{1}\left(1+\gamma_{32} P_{2}+\gamma_{31} P_{1}\right)\left(\sqrt{\gamma_{21}}-\sqrt{\gamma_{31}}\right)^{2}}{\gamma_{32} P_{2}\left(-G_{4}-G_{5}+G_{6}\right)^{2}} \geq 0 .
\end{aligned}
$$

Given that the derivative of $G\left(\rho_{z}\right)$ is negative at $\rho_{z}=0$ and non-negative at $\rho_{z}=1$, and the achievable rate is a continuous function over the correlation coefficient $\rho_{z}$, we know that there exists at least one point $\rho_{z}^{*} \in[0,1]$ such that $G^{\prime}\left(\rho_{z}^{*}\right)=0$, i.e.,

$$
-\left(G_{1} G_{5}+G_{2} G_{4}\right) \rho_{z}^{* 2}+2\left(G_{1} G_{6}+G_{3} G_{4}\right) \rho_{z}^{*}+G_{3} G_{5}-G_{2} G_{6}=0 .
$$

By solving the above equation, we have $\rho_{z}^{*}=\min \left\{\sqrt{\gamma_{31} / \gamma_{21}}, \sqrt{\gamma_{21} / \gamma_{31}}\right\}$, which is unique over [0,1]. Hence, the CF achievable rate is monotonically decreasing for $\rho_{z} \in\left[0, \rho_{z}^{*}\right]$, and monotonically increasing for $\rho_{z} \in$ $\left(\rho_{z}^{*}, 1\right]$.

As such, when we compare against the uncorrelated-noise case, the noise correlation hurts the CF scheme if $\rho_{z} \in\left(0, \rho_{z}^{\prime}\right)$, but improves the performance if $\rho_{z} \in\left(\rho_{z}^{\prime}, 1\right]$, where $\rho_{z}^{\prime} \in\left(\rho_{z}^{*}, 1\right]$ and $G\left(\rho_{z}^{\prime}\right)=G(0)$. The existence of $\rho_{z}^{\prime}$ is unique and can be calculated as

$$
\rho_{z}^{\prime}=\frac{G_{2} G_{6}-G_{3} G_{5}}{G_{1} G_{6}+G_{3} G_{4}}=\frac{2 \sqrt{\gamma_{21} \gamma_{31}}}{\gamma_{21}+\gamma_{31}} .
$$




\section{APPENDIX B}

We derive the closed-form solution of the optimal power allocation for CF under full-duplex mode. We argue that the constraint in (19) must be active at the optimal point, since for a given $P_{1}$, the relay should use all the remaining available power to maximize the achievable rate based on Theorem [5 As such, we substitute $P_{2}$ by $P_{t}-P_{1}$ in the objective function of [19].

First assuming the noises are fully-correlated, i.e., $\rho_{z}=1$ or -1 , the achievable rate of CF under full-duplex mode is $\Gamma\left(\gamma_{31} P_{1}+\gamma_{32} P_{2}\right)$. The optimization problem can be cast as:

$$
\begin{array}{cl}
\max _{P_{1}} & \left(\gamma_{31}-\gamma_{32}\right) P_{1}+\gamma_{32} P_{t} \\
\text { s.t. } & 0<P_{1} \leq P_{t} .
\end{array}
$$

If $\gamma_{31}<\gamma_{32}$, the source power $P_{1}$ should be as small as possible (but not zero due to the relay communication setup), and correspondingly, the relay power $P_{2}$ should be as large as possible given the total power constraint. Otherwise, when $\gamma_{31} \geq \gamma_{32}$, the source node should use all the power $P_{t}$ and the relay keeps silent to maximize the achievable rate.

Then we look at the case with $\rho_{z} \in(-1,1)$. Based on the alternative relay model for the CF scheme introduced in Section $\amalg$ II-E we optimize the following problem:

$$
\begin{aligned}
\max _{P_{1}, P_{2}} & \mathrm{SNR}_{\text {cf-full }} \triangleq \gamma_{31} P_{1}+\frac{\gamma_{21}^{\prime} P_{1} \gamma_{32}\left(P_{t}-P_{1}\right)}{1+\gamma_{21}^{\prime} P_{1}+\gamma_{31} P_{1}+\gamma_{32}\left(P_{t}-P_{1}\right)} \\
\text { s.t. } & 0<P_{1} \leq P_{t} .
\end{aligned}
$$

For convenience, we define the second item in the objective function as:

$$
\begin{aligned}
H & \triangleq \frac{\gamma_{21}^{\prime} P_{1} \gamma_{32}\left(P_{t}-P_{1}\right)}{1+\gamma_{21}^{\prime} P_{1}+\gamma_{31} P_{1}+\gamma_{32}\left(P_{t}-P_{1}\right)} \\
& \triangleq \frac{H_{1} P_{1}^{2}+H_{2} P_{1}}{H_{3} P_{1}+H_{4}}, \text { with } H_{1} \leq 0, H_{2} \text { and } H_{4} \geq 0
\end{aligned}
$$

The second-order derivative of the objective function is

$$
\nabla^{2} \mathrm{SNR}_{\mathrm{cf-full}}=\frac{2 H_{4}\left(H_{1} H_{4}-H_{2} H_{3}\right)}{\left(H_{3} P_{1}+H_{4}\right)^{3}} .
$$

Since the numerator

$$
H_{4} \geq 0, H_{1} H_{4}-H_{2} H_{3}=-\gamma_{21}^{\prime} \gamma_{32}-\gamma_{21}^{\prime 2} \gamma_{32} P_{t}-\gamma_{21}^{\prime} \gamma_{31} \gamma_{32} P_{t} \leq 0
$$

and the denominator

$$
\left(H_{3} P_{1}+H_{4}\right)^{3}=\left(\gamma_{21}^{\prime} P_{1}+\gamma_{31} P_{1}-\gamma_{32} P_{1}+1+\gamma_{32} P_{t}\right)^{3}>0 \text {, for } 0<P_{1} \leq P_{t} \text {, }
$$

we see that the second order derivative is non-positive

$$
\nabla^{2} \mathrm{SNR}_{\mathrm{cf-full}} \leq 0
$$


Therefore, the objective function is a concave function over the variable $P_{1}$, such that there is a global optimal solution, which is either the solution of $\nabla \mathrm{SNR}_{\mathrm{cf}-\mathrm{full}}=0$ or the boundary point $P_{t}$.

The first-order derivative over $P_{1}$ can be obtained as

$$
\nabla \mathrm{SNR}_{\text {cf-full }}=\gamma_{31}+\frac{H_{1} H_{3} P_{1}^{2}+2 H_{1} H_{4} P_{1}+H_{2} H_{4}}{\left(H_{3} P_{1}+H_{4}\right)^{2}} .
$$

When $P_{1}=0$, the first-order derivative $\nabla \mathrm{SNR}_{\mathrm{cf-full}}=\gamma_{31}+H_{2} / H_{4}>0$. If there exists an optimal solution in $\left(0, P_{t}\right)$, the first-order derivative at $P_{1}=P_{t}$ must be negative,

$$
\left.\nabla \mathrm{SNR}_{\text {cf-full }}\right|_{P_{1}=P_{t}}=\gamma_{31}-\frac{\gamma_{21}^{\prime} \gamma_{32} P_{t}}{1+\gamma_{21}^{\prime} P_{t}+\gamma_{31} P_{t}}<0,
$$

which implies that $\gamma_{21}^{\prime}\left(\gamma_{32}-\gamma_{31}\right) P_{t}>\gamma_{31}\left(1+\gamma_{31} P_{t}\right)$. Now with $\nabla \operatorname{SNR}_{\text {cf-full }}=0$, we have a second-order polynomial equation over $P_{1}$,

$$
\left(\gamma_{31} H_{3}^{2}+H_{1} H_{3}\right) P_{1}^{* 2}+\left(2 \gamma_{31} H_{3} H_{4}+2 H_{1} H_{4}\right) P_{1}^{*}+\gamma_{31} H_{4}^{2}+H_{2} H_{4}=0
$$

where only one of the two roots is in the range $\left(0, P_{t}\right)$, and can be written as the results in 20).

\section{REFERENCES}

[1] E. C. van der Meulen, "Three-terminal communication channels," Adv. Appl. Probab., vol. 3, pp. 120-154, 1971.

[2] T. M. Cover and A. El Gamal, "Capacity theorems for the relay channels," IEEE Trans. Inf. Theory, vol. 25, no. 5, pp. 572-584, Sep. 1979.

[3] A. El Gamal and M. Aref, "The capacity of the semideterministic relay channel," IEEE Trans. Inf. Theory, vol. 28, no. 3, pp. 536-536, May 1982.

[4] G. Kramer, M. Gastpar, and P. Gupta, "Cooperative strategies and capacity theorems for relay networks," IEEE Trans. Inf. Theory, vol. 51, no. 9, pp. 3037-3063, Sept. 2005.

[5] A. El Gamal and S. Zahedi, "Capacity of a class of relay channels with orthogonal components," IEEE Trans. Inf. Theory, vol. 51, no. 5, pp. 1815-1817, May 2005.

[6] T. M. Cover and Y. H. Kim, "Capacity of a class of deterministic relay channels," Proc. IEEE Int. Symp. Information Theory, Nice, France, June 24-29, 2007.

[7] A. El Gamal, M. Mohseni, and S. Zahedi, "Bounds on capacity and minimum energy-per-bit for AWGN relay channels," IEEE Trans. Inf. Theory, vol. 52, no. 4, pp. 1545-1561, Apr. 2006.

[8] A. Host-Madsen and J. Zhang, "Capacity bounds and power allocation for wireless relay channels," IEEE Trans. Inf. Theory, vol. 51, no. 6, pp. 2020-2040, Jun. 2005.

[9] J. N. Laneman, D. N. C. Tse, and G. W. Wornell, "Cooperative diversity in wireless networks: Efficient protocols and outage behavior," IEEE Trans. Inf. Theory, vol. 50, no. 12, pp. 3062-3080, Dec. 2004.

[10] J. Jiang, A. Goldsmith, and S. Cui, "Achievable rates and capacity for Gaussian relay channels with correlated noises," ISIT 2009, to appear.

[11] A. D. Wyner and J. Ziv, "The rate-distortion function for source coding with side information at the decoder," IEEE Trans. Inf. Theory, vol. 22, no. 1, pp. 1-10, Jan. 1976. 


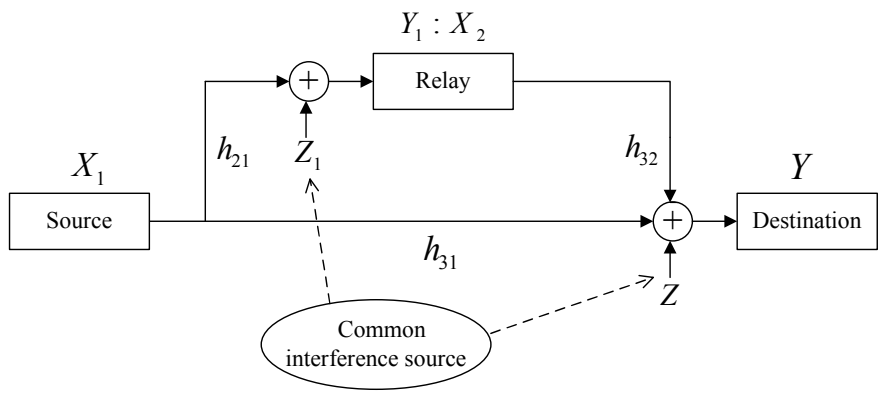

Fig. 1. The relay channel model with correlated noises at the relay and the destination.

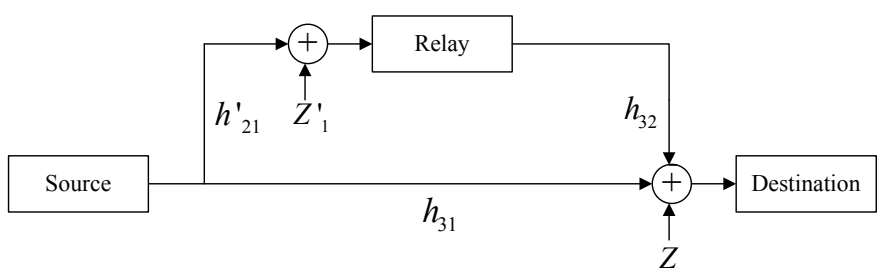

Fig. 2. The alternative relay channel model for the CF and AF schemes.

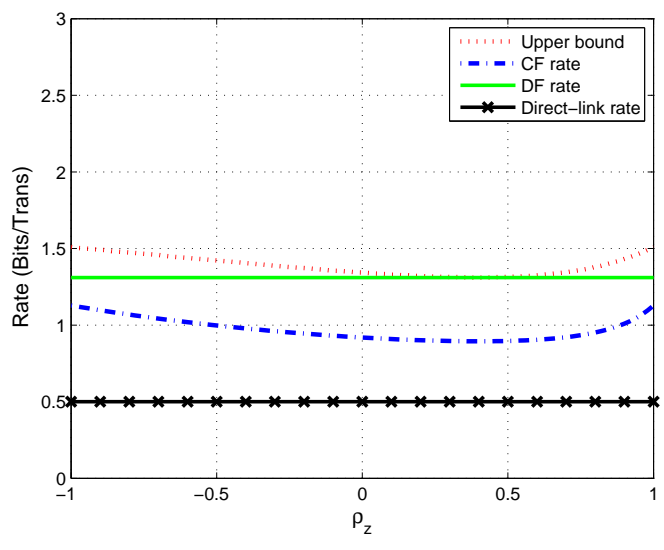

(a) Rates vs. $\rho_{z}, d=0.4$

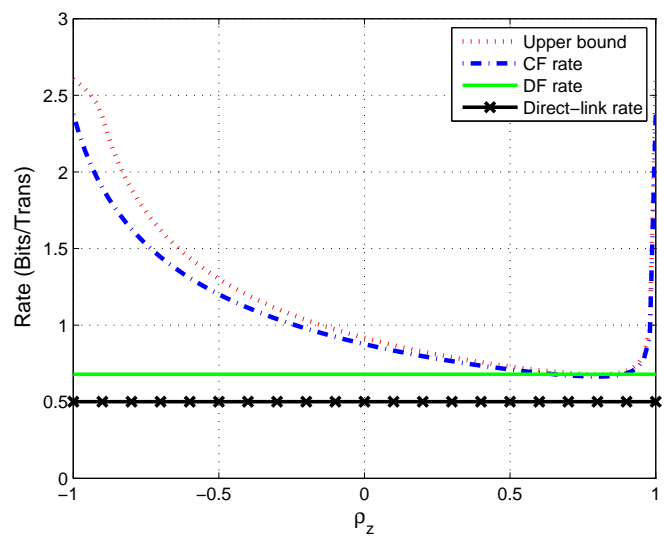

(b) Rates vs. $\rho_{z}, d=0.8$

Fig. 3. Full-duplex: Rates vs. $\rho_{z}$, with fixed power allocation. 


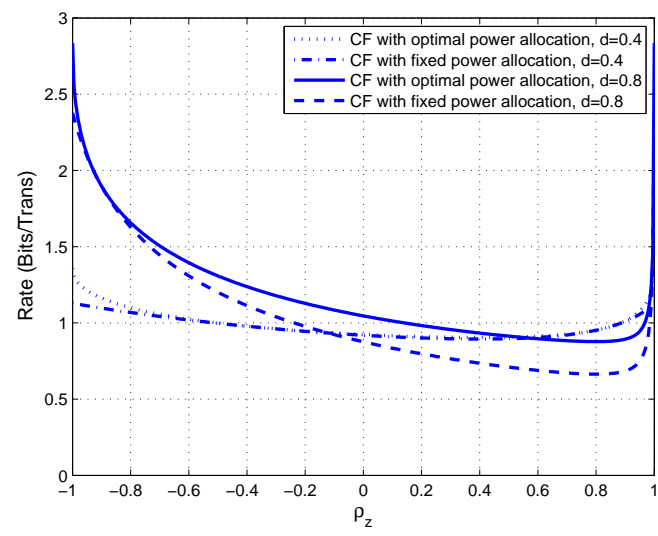

(a) $\mathrm{CF}$ rates vs. $\rho_{z}$

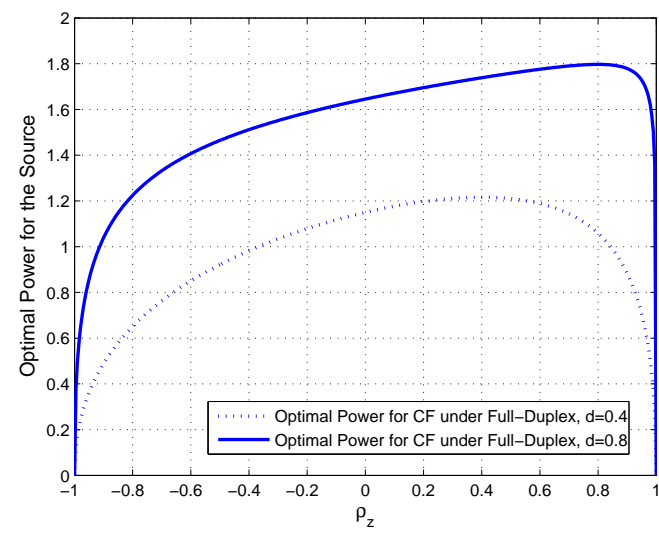

(b) Power allocation vs. $\rho_{z}$

Fig. 4. Full-duplex: (a) Rates vs. $\rho_{z}$; (b) Power Allocation vs. $\rho_{z}$.

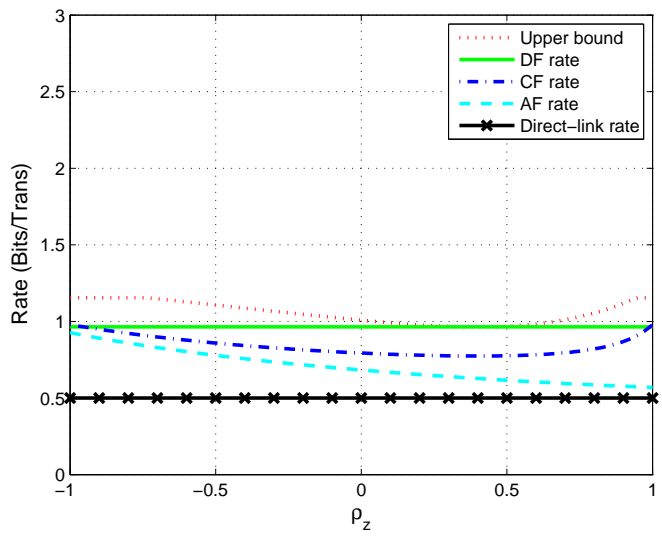

(a) Rates vs. $\rho_{z}, d=0.4$

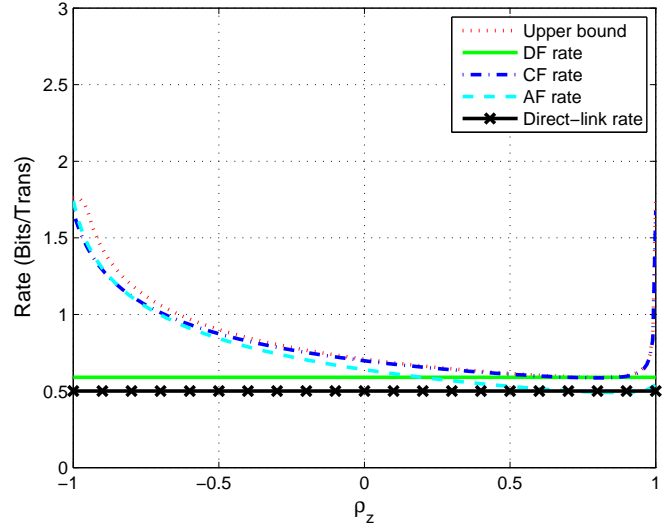

(b) Rates vs. $\rho_{z}, d=0.8$

Fig. 5. Half-duplex: Rates vs. $\rho_{z}, \alpha=0.5$, with fixed power allocation. 


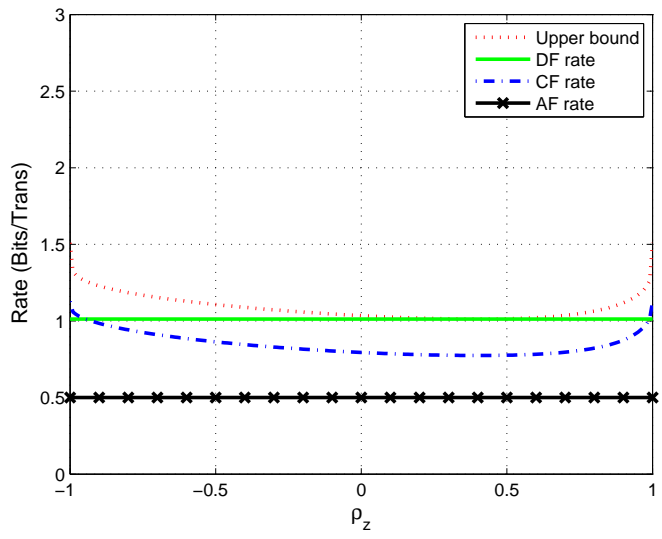

(a) Rates vs. $\rho_{z}, d=0.4$

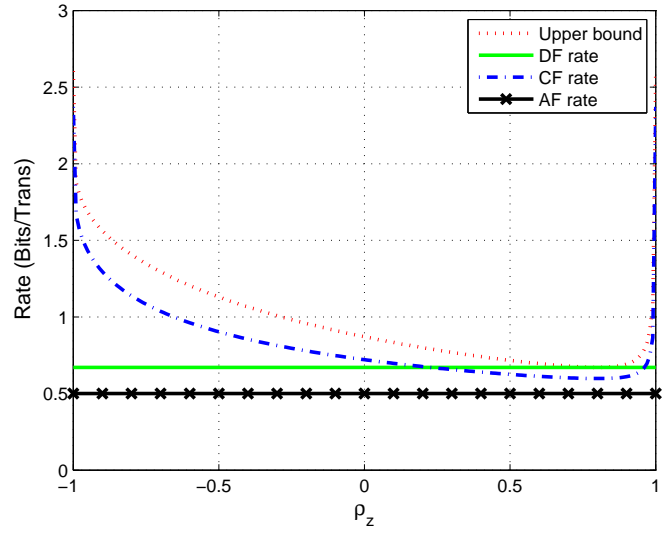

(b) Rates vs. $\rho_{z}, d=0.8$

Fig. 6. Half-duplex: Rates vs. $\rho_{z}$, optimal $\alpha$, with fixed power allocation.

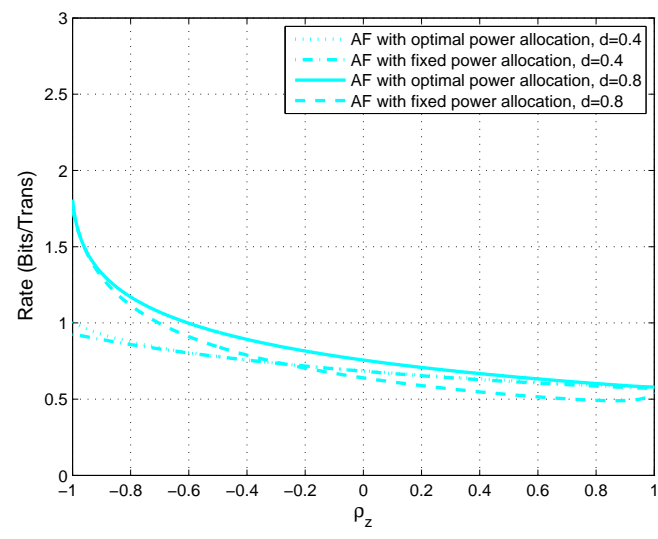

(a) AF rates vs. $\rho_{z}$

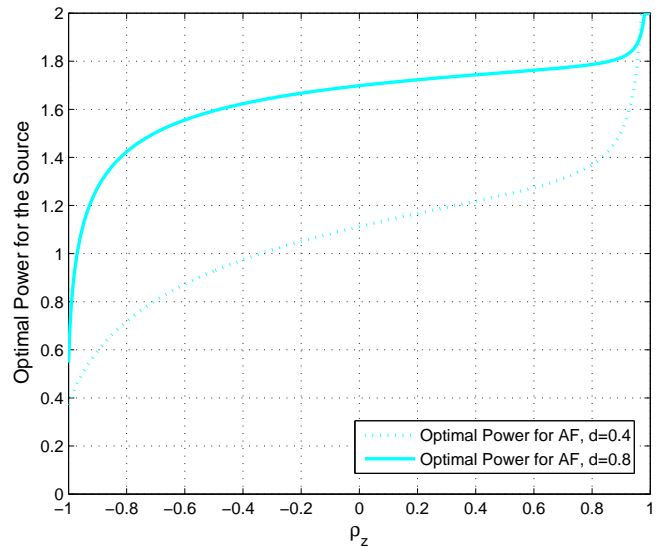

(b) Power allocation vs. $\rho_{z}$

Fig. 7. Half-duplex: (a) Rates vs. $\rho_{z}$; (b) Power Allocation vs. $\rho_{z}$. 